MATHEMATICS OF COMPUTATION

Volume 71, Number 237, Pages 297-309

S 0025-5718(01)01356-4

Article electronically published on August 2, 2001

\title{
ASYMPTOTIC PROPERTIES OF THE SPECTRAL TEST, DIAPHONY, AND RELATED QUANTITIES
}

\author{
HANNES LEEB
}

I dedicate the present work to the memory of Hans Stegbuchner

\begin{abstract}
This paper presents the limit laws of discrepancies defined via exponential sums, and algorithms (with error bounds) to approximate the corresponding distribution functions. The results cover the weighted and the nonweighted spectral test of Hellekalek and various instances of the general discrepancies of Hickernell and Hoogland and Kleiss for the exponential function system, as well as classical quantities like the spectral test, diaphony, and the Zaremba figure of merit.
\end{abstract}

\section{INTRODUCTION}

Recently, a series of papers from the Monte Carlo and quasi-Monte Carlo simulation community introduced new figures of merit for assessing random or quasirandom sequences $9,10,12,13,14$, which are more flexible alternatives to the classical star-discrepancy. The star-discrepancy gives a worst-case integration error bound - the Koksma-Hlawka inequality - when the integrand is a function of bounded variation. The new figures of merit study the worst-case and average-case integration error over different classes of functions.

For these new figures of merit (which will be simply called discrepancies), efficient computational algorithms [6, 8], estimates for particular integration sequences [5, 7, 11, and integration error bounds [12] are currently being developed (for more references, see the cited publications). Together with [15, 16, 17, 23], this paper studies the average behaviour of a (randomly selected) sequence with respect to these discrepancies. A discrepancy is used to find sequences and point-sets which behave "like random" for Monte Carlo, or which behave "more uniform than random" for quasi-Monte Carlo applications. To find sequences and point-sets with the desired properties, information on the performance of a truly random sequence or point-set with respect to the given discrepancy is required as a benchmark. This paper presents the limit laws of discrepancies defined via exponential sums, and algorithms (with error bounds) to approximate the corresponding cumulative distribution functions (cdfs). Among the yet nonunified, new discrepancies, the results

Received by the editor September 9, 1999 and, in revised form, May 5, 2000.

2000 Mathematics Subject Classification. 65D30, 11K06, 11K45, 60F05, $60 \mathrm{G} 35$.

Key words and phrases. Monte Carlo sequences, quasi-Monte Carlo sequences, equidistribution modulo one, limit distribution.

Research supported by the Austrian Science Foundation (FWF), project no. P11143-MAT.

(C)2001 American Mathematical Society 
apply to the weighted and the nonweighted spectral test of Hellekalek 9] and various instances of the general discrepancies of Hickernell [12, 13], and Hoogland and Kleiss 14 for the exponential function system, as well as classical quantities like Coveyou and MacPherson's spectral test [2], Zinterhof's diaphony [24, 25], and the Zaremba figure of merit.

The paper is organized as follows: In Section 2 the limit distributions of various discrepancies are derived. First, a class of discrepancies defined via exponential sums is studied in Theorem 1 and Corollary 1 . Examples 1 , 3 show that our approach covers various classical as well as recently proposed discrepancies, and Example 4 sketches possible applications. Second, discrepancies defined as the worst-case quadrature error over a reproducing kernel Hilbert space (as introduced by Hickernell 13|) are considered. For a particular space considered in [13, the worst-case quadrature error discrepancy and related quantities are studied in Theorem 2] and Corollary 2, For each of the discrepancies studied in Section 2, the limiting cdf turns out to be the cdf of either a sum or a maximum of a (typically infinite) number of independent, exponentially distributed random variables. As the cdfs of such sums or maxima are usually hard to compute, approximations and approximation error bounds are derived in Section 3. cf. Theorem 3 and Theorem 4. Example 5 studies the performance of these approximations for a particular discrepancy. The proofs are relegated to the Appendix.

\section{LiMiT LAWS}

Let $d \geq 1$ denote the dimension, and let $n \geq 1$ denote the sample size. For $1 \leq p \leq \infty$ and a complex-valued net $\rho=\left(\rho_{k}\right)_{k \in \mathbf{Z}^{d}}$ indexed by $\mathbf{Z}^{d}$, let $\|\rho\|_{p}=$ $\left(\sum_{k \in \mathbf{Z}^{d}}\left|\rho_{k}\right|^{p}\right)^{1 / p}$ if $p<\infty$ and $\|\rho\|_{\infty}=\sup _{k \in \mathbf{Z}^{d}}\left|\rho_{k}\right|$. Let $l_{p}^{d}(\mathbf{C})=\left\{\rho:\|\rho\|_{p}<\infty\right\}$. For two nets $\rho$ and $\nu$, denote their component-wise product by $\rho \cdot \nu=\left(\rho_{k} \nu_{k}\right)_{k \in \mathbf{Z}^{d}}$. Let $\omega=\left(\omega_{j}\right)_{j \in \mathbf{N}}$ be a sequence of points in the $d$-dimensional unit cube, i.e., $\omega_{j} \in[0,1]^{d}(j \geq 1)$. For each $k \in \mathbf{Z}^{d} \backslash\{0\}$, let

$$
S_{n, k}(\omega)=\frac{1}{n} \sum_{j=1}^{n} e^{2 \pi i k^{\prime} \omega_{j}}
$$

be the $k$-th exponential sum (of the first $n$ elements of $\omega$ ), and let $S_{n, 0}(\omega) \equiv 0$. Here and in the following, $k^{\prime} \omega_{j}$ denotes the inner product of the $d$-dimensional vectors $k$ and $\omega_{j}$. We will consider discrepancies which are functions of the net $S_{n}(\omega)=\left(S_{n, k}(\omega)\right)_{k \in \mathbf{Z}^{d}}$, i.e., discrepancies which are constructed from the ensemble $S_{n}(\omega)$ of all exponential sums $S_{n, k}(\omega)\left(k \in \mathbf{Z}^{d}\right)$.

Let $u=\left(u_{j}\right)_{j \in \mathbf{N}}$ be a sequence of independent random variables, each uniformly distributed on $[0,1]^{d}$. Throughout this section, discrepancies of the random sequence $u$ are considered. (An i.i.d. uniform sequence such as $u$ is not the only kind of random sequence occurring in the (quasi-) Monte Carlo context. Others include randomized low discrepancy sequences such as the shifted lattices of Cranley and Patterson [3] or the scrambled nets of Owen [19, 20, 21. These, however, are beyond the scope of this paper.) Corollary 1 below gives the weak limit of discrepancies of the form $\Phi\left(\rho \cdot \sqrt{n} S_{n}(u)\right)$, i.e., discrepancies constructed from exponential sums, where $\Phi$ is an appropriate function and $\rho \cdot \sqrt{n} S_{n}(u)=\left(\rho_{k} \sqrt{n} S_{n, k}(u)\right)_{k \in \mathbf{Z}^{d}}$ is the ensemble of the weighted exponential sums with appropriate weights $\rho_{k}\left(k \in \mathbf{Z}^{d}\right)$. Write $S_{n}$ and $S_{n, k}$ as shorthand notation for $S_{n}(u)$ and $S_{n, k}(u)$, respectively. To describe the weak limits, let $T=\left(T_{k}\right)_{k \in \mathbf{Z}^{d}}$ be such that $T_{0} \equiv 0$ and, for $k \neq 0$, 
$T_{k}=M_{k}+i N_{k}$, where $M_{k}$ and $N_{k}$ are i.i.d. $N(0,1 / 2)$; finally, let the correlation of $T_{k}$ and $T_{l}$ be such that $T_{k}$ is independent of $T_{l}$ if $k \neq \pm l$, and such that $T_{k}=\bar{T}_{-k}$.

Theorem 1. If $\rho \in l_{p}^{d}(\mathbf{C})(1 \leq p<\infty)$, then $\rho \cdot \sqrt{n} S_{n}$ converges weakly to $\rho \cdot T$ in $l_{p}^{d}(\mathbf{C})$.

Corollary 1. Let $\Phi$ be a continuous function on $l_{p}^{d}(\mathbf{C})$ and $\rho \in l_{p}^{d}(\mathbf{C})(1 \leq p<\infty)$. From Theorem 1 and the Continuous Mapping Theorem, we immediately obtain that $\Phi\left(\rho \cdot \sqrt{n} S_{n}\right)$ converges weakly to $\Phi(\rho \cdot T)$. If $\Phi(\rho \cdot T)$ is real-valued and if the cdf of $\Phi(\rho \cdot T)$ is continuous (as is the case in the examples considered below), then $P\left(\Phi\left(\rho \cdot \sqrt{n} S_{n}\right) \leq x\right)$ converges to $P(\Phi(\rho \cdot T) \leq x)$ uniformly in $x$ by Polya's Theorem.

Various discrepancies have the form $\Phi\left(\rho \cdot \sqrt{n} S_{n}\right)$, as we shall show in the examples below. The (asymptotic) distribution of a discrepancy can be used to answer some questions of interest for (quasi-) Monte Carlo use; given a particular sequence $\omega$ and its discrepancy $\Phi\left(\rho \cdot \sqrt{n} S_{n}(\omega)\right)$, one may ask, say: Is $\omega$ better (with respect to that discrepancy) than an average random sequence, i.e., is $\Phi\left(\rho \cdot \sqrt{n} S_{n}(\omega)\right)<$ $E\left(\Phi\left(\rho \cdot \sqrt{n} S_{n}\right)\right)$ ? Is $\omega$ likely to outperform a randomly selected sequence, i.e., is $P\left(\Phi\left(\rho \cdot \sqrt{n} S_{n}(\omega)\right)<\Phi\left(\rho \cdot \sqrt{n} S_{n}\right)\right)>1 / 2$ ? Does $\omega$ mimic the behaviour of a truly random sequence; e.g., does $\Phi\left(\rho \cdot \sqrt{n} S_{n}(\omega)\right) \in[a, b]$ hold (where $a$ is the $\alpha / 2$ and $b$ the $1-\alpha / 2$ quantile of the discrepancy's distribution)? What is the 'natural' scale of the discrepancy, i.e., what is the value of $\operatorname{Var}\left(\Phi\left(\rho \cdot \sqrt{n} S_{n}\right)\right)$ ? A sketch of how the results in this paper can be used to address these questions is given in Example 4

Example 1. (The weighted spectral test for the exponential function system, and the diaphony): If $\rho \in l_{2}^{d}(\mathbf{C})$ is real- and positive-valued, then $\left\|\rho \cdot S_{n}\right\|_{2}$ is just the weighted spectral test introduced by Hellekalek [9] or the Fourier discrepancy considered by Hoogland and Kleiss [14]. Since the norm is continuous on $l_{2}^{d}(\mathbf{C})$, the weak limit of $\left\|\rho \cdot \sqrt{n} S_{n}\right\|_{2}$ is $\|\rho \cdot T\|_{2}$, the square root of a quadratic form in normal random variables. In particular, for $\eta=\left(\eta_{k}\right)_{k \in \mathbf{Z}^{d}} \in l_{2}^{d}(\mathbf{C})$ defined by $\eta_{k}=\prod_{l=1}^{d} \eta_{k}^{(l)}$, $\eta_{k}^{(l)}=i / k^{(l)}$ if $k^{(l)} \neq 0, \eta_{k}^{(l)}=1$ if $k^{(l)}=0$, for $k=\left(k^{(1)}, \ldots, k^{(d)}\right)^{\prime} \in \mathbf{Z}^{d} \backslash\{0\}$, and $\eta_{(0, \ldots, 0)^{\prime}}=0,\left\|\eta \cdot S_{n}\right\|_{2}$ is the diaphony introduced by Zinterhof [24].

Example 2. (The spectral test for the exponential function system, the traditional spectral test, and the Zaremba figure of merit): If $\rho \in l_{p}^{d}(\mathbf{C})(1 \leq p<\infty)$ is real- and positive-valued, then $\sigma_{n}(\rho)=\left\|\rho \cdot S_{n}\right\|_{\infty}$ is the spectral test of Hellekalek [9]. The weak limit of $\sqrt{n} \sigma_{n}(\rho)$ is $\|\rho \cdot T\|_{\infty}$, the square root of a maximum of independent, exponentially distributed random variables (because $T_{k}=\bar{T}_{-k}$; cf. (6) ). For $\gamma=\left(\gamma_{k}\right)_{k \in \mathbf{Z}^{d}}$ defined by $\gamma_{k}=1 /\|k\|_{2}$ for $k \neq 0$ and $\gamma_{0}=0, \sigma_{n}(\gamma)$ is the traditional spectral test of Coveyou and MacPherson 2], as pointed out by Hellekalek 9. (Here and in the following, the standard Euclidean norm on $\mathbf{R}^{d}$ is denoted by $\|\cdot\|_{2}$.) Also note that for the particular choice of $\eta$ as in Example $\left\|\eta \cdot S_{n}(\omega)\right\|_{\infty}$ coincides with the Zaremba figure of merit if the points of $\omega$ form a grid in $[0,1]^{d}$.

Example 3. (Discrepancies as worst-case quadrature error bounds): This notion of discrepancy, which gives a worst-case quadrature error bound over a certain class of functions, was introduced by Hickernell [12, 13]; with Theorem 1, we obtain the weak limit for several instances of this concept. For $\eta$ as in Example 1) set $\eta^{x}=\left(\eta_{k}^{x}\right)_{k \in \mathbf{Z}^{d}}$; then $\left\|\eta^{\alpha / 2} \cdot S_{n}\right\|_{2}$ is, except for a constant shift, the quantity in (3.9) of [13], and $\left\|\eta^{\alpha} \cdot S_{n}\right\|_{p}$ is that in (3.16) of [13]. Adapting the vector $\eta$, we also 
obtain the weak limits of the ANOVA decomposition discrepancy (4.5) of [13] and the weighted generalization (4.11) of [13].

Example 4. As outlined in the discussion following Corollary 1 the results in this paper can be used to compare a particular fixed (quasi-) Monte Carlo sequence $\omega$ with an i.i.d. uniform random sequence $u$ with respect to, say, the diaphony, i.e., with respect to $\left\|\eta \cdot \sqrt{n} S_{n}(\omega)\right\|_{2}$ (cf. Examples 1 and 3). Consider first the case where $\omega$ is intended for quasi-Monte Carlo use. Since the limiting cdf of $\left\|\eta \cdot \sqrt{n} S_{n}\right\|_{2}^{2}$ is continuous, we obtain from Corollary 1 that

$$
P\left(\left\|\eta \cdot \sqrt{n} S_{n}(\omega)\right\|_{2}<\left\|\eta \cdot \sqrt{n} S_{n}\right\|_{2}\right) \longrightarrow 1,
$$

i.e., the probability of $\omega$ outperforming a randomly selected sequence converges to one, as $n \rightarrow \infty$, if and only if $\left\|\eta \cdot \sqrt{n} S_{n}(\omega)\right\|_{2} \rightarrow 0$ as $n \rightarrow \infty$. For a finite-sample comparison, we note that elementary calculations give the finite-sample moments

$$
E\left(\left\|\eta \cdot \sqrt{n} S_{n}\right\|_{2}^{2}\right)=\left(1+\pi^{2} / 3\right)^{d}-1
$$

and

$$
\operatorname{Var}\left(\left\|\eta \cdot \sqrt{n} S_{n}\right\|_{2}^{2}\right)=2\left(\left(1+\pi^{4} / 45\right)^{d}-1\right)(n-1) / n,
$$

while the large-sample moments are $\mu=E\left(\|\eta \cdot T\|_{2}^{2}\right)=\left(1+\pi^{2} / 3\right)^{d}-1$ and $\sigma^{2}=$ $\operatorname{Var}\left(\|\eta \cdot T\|_{2}^{2}\right)=2\left(\left(1+\pi^{4} / 45\right)^{d}-1\right)$. Moreover, Corollary 1 together with Theorem[3 below gives an approximation for $P\left(\left\|\eta \cdot \sqrt{n} S_{n}(\omega)\right\|_{2}<\left\|\eta \cdot \sqrt{n} S_{n}\right\|_{2}\right)$, namely $1-$ $P\left(R \leq\left(2 \nu / \sigma^{2}\right)^{1 / 2}\left(\left\|\eta \cdot \sqrt{n} S_{n}(\omega)\right\|_{2}^{2}-\mu\right)+\nu\right)$, where $R$ is gamma-distributed with mean $\nu$ and variance $2 \nu=2\left(\sum_{k}\left|\eta_{k}\right|^{4}\right)^{3}\left(\sum_{k}\left|\eta_{k}\right|^{6}\right)^{-2}$. For the diaphony, elementary calculations show that

$$
\nu=\left(\left(1+\pi^{4} / 45\right)^{d}-1\right)^{3}\left(\left(1+2 \pi^{6} / 945\right)^{d}-1\right)^{-2}
$$

(see Example 5 concerning the accuracy of this approximation). For the actual value of the diaphony of $\omega$, i.e., for $\left\|\eta \cdot \sqrt{n} S_{n}(\omega)\right\|_{2}$, either estimates such as given in [5, 7, 11] or direct computation may be employed. (Concerning the latter, we note that the algorithm of Heinrich [8] can be adapted to the diaphony; this algorithm requires $O\left(n(\log n)^{d}\right)$ operations.) For the case where $\omega$ is intended for Monte Carlo use, the above observations can be used to construct various tests on the hypothesis that $\omega$ is a realization of an i.i.d. uniform sequence $u$.

In Hickernell's concept of discrepancy as worst-case quadrature error for a given reproducing kernel Hilbert space [13, the discrepancy is expressed as the norm of a particular function called the 'representer' from that space, which depends on the sequence of points $u$. For a particular instance of the spaces considered in 13, we derive the weak limit of the representer as a random function below. From this, the corresponding limit of the discrepancy and of any other continuous function of the representer follows immediately. Let $B_{1}(x)=(x \bmod 1)-1 / 2$ be the periodic extension of the first Bernoulli polynomial on $[0,1)$, and, for $t=\left(t^{(1)}, \ldots, t^{(d)}\right)^{\prime} \in$ $\mathbf{R}^{d}$, let

$$
\begin{aligned}
& f(t)=\prod_{l=1}^{d}\left(2 \pi B_{1}\left(t^{(l)}\right)+1\right)-1 \quad \text { and } \\
& g(t)=\int_{[0,1]^{d}} f(x) f(x-t) d x=\prod_{l=1}^{d}\left(h\left(t^{(l)}\right)+1\right)-1,
\end{aligned}
$$


where $d x$ denotes integration w.r.t. Lebesgue measure and where

$$
h(x)=4 \pi^{2} \int_{0}^{1} B_{1}(z) B_{1}(z-x) d z=2 \pi^{2}\left(x^{2}-x+1 / 6\right) .
$$

Then

$$
X_{n}(t, u)=\frac{1}{\sqrt{n}} \sum_{j=1}^{n} f\left(u_{j}-t\right)
$$

defines a real-valued, random function $X_{n}$ on the $d$-dimensional unit cube. Note that the Fourier-coefficients of $f$ are just the values of $\eta$ from Example 1 and hence $\left\|\eta \cdot \sqrt{n} S_{n}\right\|_{2}=\left\|X_{n}\right\|_{2}$.

\section{Theorem 2.}

1. The sequence $\left(X_{n}\right)_{n \in \mathbf{N}}$ converges weakly to a continuous Gaussian process $X$ with $E(X(t))=0, E(X(s) X(t))=g(t-s)$ in the space $C\left([0,1]^{d}\right)$ of realvalued continuous functions on $[0,1]^{d}$.

2. The limit process $X$ has the representation $X(t)=\sum_{k \in \mathbf{Z}^{d}} \eta_{k} T_{k} e^{2 \pi i k^{\prime} t}$, where the sequence converges uniformly in $t$ with probability one.

Corollary 2. Let $\Phi$ be a continuous function on $C\left([0,1]^{d}\right)$. From Theorem 2 , we can conclude that $\Phi\left(X_{n}\right)$ converges weakly to $\Phi(X)$, and that the distribution of $\Phi(X)$ is just that of $\lim _{K} \Phi\left(\sum_{k \in \mathbf{Z}^{d},\|k\|_{2} \leq K} \eta_{k} T_{k} e^{2 \pi i k^{\prime} t}\right)$. In particular, the convolution operator is continuous on $C\left([0,1]^{d}\right)$.

Remark 1. The ensemble of exponential sums, i.e., $S_{n}(\omega)$, was chosen in this section because many discrepancies in use today can be expressed by $S_{n}(\omega)$. Yet, other systems of orthonormal functions might be used to construct discrepancies, like the dyadic diaphony 10 which is based on the system of Walsh functions of base 2 . Inspection of the proof shows that Theorem 1 readily adapts to this case (for an appropriate choice of $T$ ). Extensions of Theorem 2 to other reproducing kernel Hilbert spaces will be discussed elsewhere.

\section{Approximations to the Limiting Distribution FUnCtions}

In the following, approximations to the limiting cdfs of the discrepancies encountered in the previous section are considered, i.e., approximations to the cdfs of $\|\rho \cdot T\|_{2}$ and $\|\rho \cdot T\|_{\infty}$ for appropriate weights $\rho$. Recall that the correlation of $T_{k}$ and $T_{l}$ is such that $\left|T_{k}\right|$ is independent of $\left|T_{l}\right|$ for $k \neq \pm l$ and $\left|T_{k}\right|=\left|T_{-k}\right|$. Let $I_{+}=\left\{k \in \mathbf{Z}^{d} \backslash\{0\}: k=\left(0, \ldots, 0, k_{l}, \ldots, k_{d}\right)^{\prime}, k_{l}>0,1 \leq l \leq d\right\}$ be the set of those $k \in \mathbf{Z}^{d} \backslash\{0\}$ for which the first nonzero coordinate is positive. Observing that $\mathbf{Z}^{d} \backslash\{0\}=I_{+} \cup\left\{-k: k \in I_{+}\right\}$, we obtain that

$$
\begin{aligned}
\|\rho \cdot T\|_{2}^{2} & =\sum_{k \in I_{+}}\left(\left|\rho_{k}\right|^{2}+\left|\rho_{-k}\right|^{2}\right)\left(M_{k}^{2}+N_{k}^{2}\right), \\
\|\rho \cdot T\|_{\infty}^{2} & =\sup _{k \in I_{+}} \max \left\{\left|\rho_{k}\right|^{2},\left|\rho_{-k}\right|^{2}\right\}\left(M_{k}^{2}+N_{k}^{2}\right) .
\end{aligned}
$$

Hence, the cdf of (5) and (6) is that of a sum and a maximum, respectively, of independent exponentially distributed random variables. (Whenever $\left|\rho_{k}\right|=\left|\rho_{-k}\right|$, as in some of the examples, this representation is further simplified.)

In case $d=1$ the cdf of $\|\eta \cdot T\|_{2}^{2}$ from Example[1, i.e., the limiting distribution of the diaphony in one dimension, is up to trivial scaling the Kolmogorov-Smirnov 


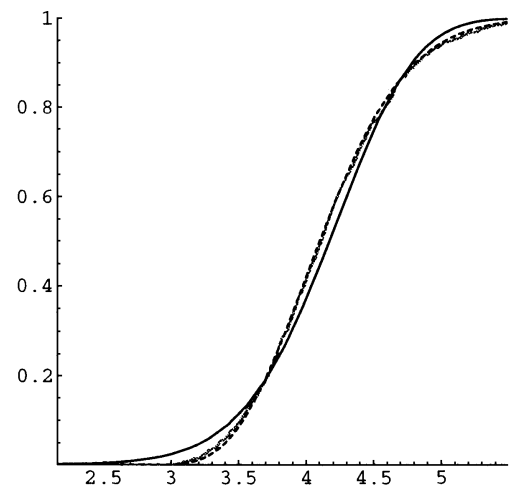

Figure 1. Empirical cdf of $\left\|\eta \cdot \sqrt{n} S_{n}\right\|_{2}$ with $n=128$ and $d=2$ obtained from 1024 repetitions (dotted line), gamma approximation (dashed line), and normal approximation (solid line).

distribution [16, for which a rapidly converging series-representation is known. In higher dimensions, the cdf of $\|\rho \cdot T\|_{2}^{2}$ (properly scaled and centered) approaches a normal cdf $[16,23$. While the normal approximation is satisfactory in high dimensions, the gamma approximation given below performs even better. To simplify notation for the rest of this section, we consider real-valued, nonnegative weights $\varrho=\left(\varrho_{k}\right)_{k \in \mathbf{N}}$, i.i.d. standard normal variates $N=\left(N_{k}\right)_{k \in \mathbf{N}}$, and i.i.d. standard exponentially distributed random variables $C=\left(C_{k}\right)_{k \in \mathbf{N}}$ (i.e., $E\left(C_{k}\right)=1, k \geq 1$ ). The cases (5) and (6) reduce to $\|\varrho \cdot C\|_{2}^{2}$ and $\|\varrho \cdot C\|_{\infty}$ by relabelling and appropriate choice of $\varrho$.

Theorem 3. Let $\sum_{k} \varrho^{2}<\infty$. Then

$$
\mu=E\left(\|\varrho \cdot N\|_{2}^{2}\right)=\sum_{k} \varrho_{k}^{2}, \quad \sigma^{2}=\operatorname{Var}\left(\|\varrho \cdot N\|_{2}^{2}\right)=2 \sum_{k} \varrho_{k}^{4} .
$$

If $R$ is gamma-distributed with $E(R)=\nu=\left(\sum_{k} \varrho_{k}^{4}\right)^{3}\left(\sum_{k} \varrho_{k}^{6}\right)^{-2}$ and $\operatorname{Var}(R)=2 \nu$, then

$$
\left|P\left(\frac{\|\varrho \cdot N\|_{2}^{2}-\mu}{\sigma} \leq t\right)-P\left(\frac{R-\nu}{\sqrt{2 \nu}} \leq t\right)\right| \leq B,
$$

uniformly in $t$, where the constant $B$, given in (11), is explicitly computable from $\varrho$.

Since the cdf of $\|\varrho \cdot C\|_{2}$ equals that of $\|\vartheta \cdot N\|_{2}$ if $\vartheta_{2 j}=\vartheta_{2 j-1}=\varrho_{j} / 2$ for $j \geq 1$, Theorem 3 also gives an approximation to the cdf of the quantity in (5).

Example 5. Consider the particular weights $\eta$ used in Examples 1 and 3 above. When we approximate the limiting cdf of the diaphony, i.e., the cdf of $\|\eta \cdot T\|_{2}^{2}$, by a normal law [16, 23], we can derive an error bound similar to that of Theorem 3 However, we found the normal approximation less satisfactory than the gamma approximation in moderate dimensions, and Figure 1 seems to support this. To apply Theorem [3, i.e., to compute the error bound $B$ as given in (11), we note for the constants occurring in $B$ that the sum of (powers of) $\left|\eta_{k}\right|^{2}$ can be easily computed, and we have $\varrho_{*}^{2}=1$ and $K=3^{d}-1$ in dimension $d$. In Table 1 , we give the error bounds for approximation of the cdf of $\|\eta \cdot T\|_{2}^{2}$ for various dimensions 
TABLE 1. Error bound from Theorem 3 for approximating the cdf of $\|\eta \cdot T\|_{2}^{2}$ by a gamma distribution's cdf. The shown dimensions $d$ are those where the accuracy increases by an order of magnitude.

\begin{tabular}{r|l}
$d$ & approximation error bound \\
\hline 2 & 0.150321 \\
3 & 0.0441517 \\
5 & 0.00292051 \\
6 & 0.000835115 \\
8 & 0.0000726281 \\
10 & 0.0000063662
\end{tabular}

$d$. Together with Corollary 1 Theorem 3 also gives an approximation to the finitesample cdf of the diaphony, uniformly in $t$. The quality of this approximation, however, depends on the speed of convergence of the cdf of $\left\|\eta \cdot \sqrt{n} S_{n}\right\|_{2}^{2}$ to the cdf of $\|\eta \cdot T\|_{2}^{2}$. In two dimensions, i.e., for $d=2$, Figure 1 suggests that these cdfs are fairly close for $n \geq 128$. The rate of convergence for other values of $d \geq 1$ and related topics are subject to further research.

Remark 2. Theorem 3 is motivated by a result of Buckley and Eagleson 1, who consider the case of finitely many nonzero $\varrho_{k}$. We extend the result to infinitely many nonzero $\varrho_{k}$ and improve the error bound.

Remark 3. If the weights $\varrho$ are such that the approximation from Theorem 3 is unsatisfactory (which happens, say, for the dyadic diaphony [10] in dimension 2; cf. Remark (1), the cdf of $\|\varrho \cdot N\|_{2}^{2}$ can, alternatively, be approximated by that of $\left\|\varrho^{(m)} \cdot N\right\|_{2}^{2}$ for large $m$, where $\varrho^{(m)}=\left(\varrho_{k}^{(m)}\right)_{k \in \mathbf{N}}$ with $\varrho_{k}^{(m)}=\varrho_{k}$ for $k \leq m$ and $\varrho_{k}^{(m)}=0$ otherwise. This follows from Corollary 1 and a standard uniform approximation argument [22, p.70]. The cdf of the finite sum of squares of independent normal variates $\left\|\varrho^{(m)} \cdot N\right\|_{2}^{2}$ can be computed with the algorithm of Farebrother [4].

Proceeding as in Remark 3, we also obtain an approximation to the cdf of $\|\varrho \cdot C\|_{\infty}^{2}$ by the cdf of a maximum of finitely many independent exponentially distributed random variables, which is directly computable. As before, let $\varrho^{(m)}=$ $\left(\varrho_{k}^{(m)}\right)_{k \in \mathbf{N}}$ with $\varrho_{k}^{(m)}=\varrho_{k}$ for $k \leq m$ and $\varrho_{k}^{(m)}=0$ otherwise.

Theorem 4. Let $m \geq p$ be positive integers, and set $\alpha_{p}=E\left(C_{1}^{p}\right)$. If $\sum_{k} \varrho_{k}^{p}<\infty$, then

$$
\begin{aligned}
\mid P\left(\|\varrho \cdot C\|_{\infty} \leq t\right)- & P\left(\left\|\varrho^{(m)} \cdot C\right\|_{\infty} \leq t\right) \mid \\
\leq & \alpha_{p}\left(\sum_{k>m}\left|\varrho_{k}\right|^{p}\right) \min \left\{\prod_{l=1}^{p} \frac{1}{\left|\varrho_{l}\right|}, t^{-p}\right\},
\end{aligned}
$$

uniformly in $t>0$.

\section{Appendix: Proofs}

Proof of Theorem 1 . Let $\{x\}$ denote the fractional part of $x$. Since the Lebesgue measure $\lambda$ is the Haar measure on the torus, it follows that, for $k \in \mathbf{Z}^{d} \backslash\{0\}$, $\left(\left\{k^{\prime} u_{j}\right\}\right)_{j \in \mathbf{N}}$ is a sequence of independent random variables, each uniformly distributed on $[0,1]$. Since $\exp \left(2 \pi i k^{\prime} x\right)=\exp \left(2 \pi i\left\{k^{\prime} x\right\}\right)$, the Central Limit Theorem 
immediately gives that $\sqrt{n} S_{n, k}$ converges weakly to $T_{k}$ for fixed $k \in \mathbf{Z}^{d}$. By orthogonality of the exponential function system, we obtain, for each $m \in \mathbf{N}$ and each set of indices $\left\{k_{1}, \ldots, k_{m}\right\} \subset \mathbf{Z}^{d}$, that the vector $\sqrt{n}\left(S_{n, k_{1}}, \ldots, S_{n, k_{m}}\right)$ converges weakly to $\left(T_{k_{1}}, \ldots, T_{k_{m}}\right)$. For $m \in \mathbf{N}$, let $\rho^{(m)}=\left(\rho_{k}^{(m)}\right)_{k \in \mathbf{Z}^{d}}$ with $\rho_{k}^{(m)}=\rho_{k}$ if $\|k\|_{2} \leq m$ and $\rho_{k}^{(m)}=0$ otherwise. Since, for fixed $m, \rho^{(m)} \cdot \sqrt{n} S_{n}$ converges weakly to $\rho^{(m)} \cdot T$, weak convergence of $\rho \cdot \sqrt{n} S_{n}$ to $\rho \cdot T$ in $l_{p}^{d}(\mathbf{C})$ follows from a standard uniform approximation argument [22, p.70], if $\rho \cdot T$ and $\rho \cdot \sqrt{n} S_{n}$ are random elements in $l_{p}^{d}(\mathbf{C})$, and if, for each $\epsilon>0$,

$$
\begin{aligned}
\lim _{m} P\left(\left\|\rho \cdot T-\rho^{(m)} \cdot T\right\|_{p} \geq \epsilon\right) & =0, \\
\lim _{m} \sup _{n} P\left(\left\|\rho \cdot \sqrt{n} S_{n}-\rho^{(m)} \cdot \sqrt{n} S_{n}\right\|_{p} \geq \epsilon\right) & =0 .
\end{aligned}
$$

To show that $\rho \cdot T \in l_{p}^{d}(\mathbf{C})$, we remark that we shall prove (7) using Chebyshev's inequality; i.e., we shall show that $E\left\|\rho^{(m)} \cdot T-\rho \cdot T\right\|_{p} \leq K<\infty$ for each $m$. Since $E\left\|\rho^{(m)} \cdot T\right\|_{p}<\infty$, it follows that $E\|\rho \cdot T\|_{p}<\infty$ and hence $\|\rho \cdot T\|_{p}<\infty$ with probability one. Hence, we may adjust the probability space such that $\rho \cdot T \in l_{p}^{d}(\mathbf{C})$. The same argument gives $\rho \cdot \sqrt{n} S_{n} \in l_{p}^{d}(\mathbf{C})$. For (7), set $\mu_{p}=E\left|T_{k}\right|^{p}$. Since $\left|T_{k}\right|^{2}$ is exponentially distributed, $\mu_{p}<\infty$, and we obtain

$$
P\left(\left\|\rho \cdot T-\rho^{(m)} \cdot T\right\|_{p} \geq \epsilon\right) \leq \frac{\mu_{p}}{\epsilon^{p}} \sum_{\substack{k \in \mathbf{Z}^{d} \\\|k\|_{2}>m}}\left|\rho_{k}\right|^{p},
$$

which gives (7). Similarly, for (8), we obtain

$$
P\left(\left\|\rho \cdot \sqrt{n} S_{n}-\rho^{(m)} \cdot \sqrt{n} S_{n}\right\|_{p} \geq \epsilon\right) \leq \frac{1}{\epsilon^{p}} \sum_{\substack{k \in \mathbf{Z}^{d} \\\|k\|_{2}>m}}\left|\rho_{k}\right|^{p} E\left(\left|\sqrt{n} S_{n, k}\right|^{p}\right) .
$$

Since, for each fixed $k \neq 0,\left(\left\{k^{\prime} u_{j}\right\}\right)_{j \in \mathbf{N}}$ is distributed as an independent sequence $\left(v_{j}\right)_{j \in \mathbf{N}}$ of random variables uniform on $[0,1]$, we conclude that $\mu_{p, n}=$ $E\left(\left|\sqrt{n} S_{n, k}\right|^{p}\right)$ is independent of $k$. The proof is complete if $\mu_{p, n}$ is uniformly bounded in $n$. From Jensen's inequality, we see that $\mu_{p, n} \leq\left(\mu_{q, n}\right)^{p / q}$ whenever $p \leq q$. Therefore, it is sufficient to show that $\mu_{4 a, n}$ is uniformly bounded in $n$, where $a$ is a positive integer. Now

$$
\begin{aligned}
\mu_{4 a, n} & =E\left(\left(\frac{1}{\sqrt{n}} \sum_{j=1}^{n} \cos 2 \pi v_{j}\right)^{2}+\left(\frac{1}{\sqrt{n}} \sum_{j=1}^{n} \sin 2 \pi v_{j}\right)^{2}\right)^{2 a} \\
& =\sum_{l=0}^{2 a}\left(\begin{array}{c}
2 a \\
l
\end{array}\right) E\left(\left(\frac{1}{\sqrt{n}} \sum_{j=1}^{n} \cos 2 \pi v_{j}\right)^{2 l}\left(\frac{1}{\sqrt{n}} \sum_{j=1}^{n} \sin 2 \pi v_{j}\right)^{2(2 a-l)}\right) .
\end{aligned}
$$

With Hoelder's inequality, this is uniformly bounded in $n$, if, for each positive integer $b, E\left(\left(\frac{1}{\sqrt{n}} \sum_{j=1}^{n} \cos 2 \pi v_{j}\right)^{2 b}\right)=E\left(\left(\frac{1}{\sqrt{n}} \sum_{j=1}^{n} \sin 2 \pi v_{j}\right)^{2 b}\right)$ is uniformly bounded 
in $n$. The first of these expectations is the sum of $n^{2 b}$ terms, each of the form

$$
\frac{1}{n^{b}} E\left(\prod_{l=1}^{2 b} \cos 2 \pi v_{j_{l}}\right)
$$

where $\left(j_{1}, \ldots, j_{2 b}\right) \in\{1, \ldots, n\}^{2 b}$. But whenever an index, say, $j_{1}$ is different from the others, the value of (91) is zero by independence of the $v_{i}$. Conversely, (91) is nonzero only for at least pairwise equal indices. Since this is possible for at most $C_{b} n^{b}$ terms, where $C_{b}$ is a finite combinatorial constant depending only on $b$, we obtain $E\left(\left(\frac{1}{\sqrt{n}} \sum_{j=1}^{n} \cos 2 \pi v_{j}\right)^{2 b}\right) \leq C_{b}$.

Proof of Theorem Q As the classical empirical process is defined by the class of all indicator functions on rectangles with one vertex in the origin, the process $X_{n}$ is defined by the class

$$
\mathcal{F}=\left\{f(\cdot-t): t \in[0,1]^{d}\right\}
$$

of functions indexed by $t \in[0,1]^{d}$. Limit theorems for an empirical process defined by a class of functions are trivial for a finite class and still hold if the class is not too large in a certain sense [22]. We show that this is true for $\mathcal{F}$. Equip $\mathcal{F}$ with the $L^{2}(\lambda)$-norm, where $\lambda$ is the Lebesgue measure on $[0,1]^{d}$. It is easy to verify that $\mathcal{F}$ is a set of measurable, uniformly bounded and, in the sense of 22 . p.196], permissible functions. Since $\left\|f(\cdot-t)-f\left(\cdot-t_{0}\right)\right\|_{2}^{2}=2\left(g(0)-g\left(t-t_{0}\right)\right)$, and $g$ is continuous at $0, \mathcal{F}$ is also totally bounded with respect to the $L^{2}(\lambda)$ norm. Let us first show that the family of graphs $\mathcal{G}=\left\{G_{f_{t}}: f_{t} \in \mathcal{F}\right\}$, where $G_{f_{t}}=\left\{(s, z): s \in[0,1]^{d}, z \in \mathbf{R}, 0 \leq z \leq f_{t}(s)\right.$ or $\left.f_{t}(s) \leq z \leq 0\right\}$, is a polynomial class in the sense of [22, p.17, Definition 13]. By Lemma 28 of [22, p.30], the set $\mathcal{P}$ of graphs of polynomials on $[0,1]^{d}$ of degree at most one in each coordinate is a polynomial class. Now each $t \in[0,1]^{d}$ partitions the unit cube in $2^{d}$ quadrants $Q_{i, t}$ $\left(i=1, \ldots, 2^{d}\right)$, and on each quadrant, $f(\cdot-t) \in \mathcal{F}$ is a polynomial of degree one in each coordinate. Since the quadrants themselves form a polynomial class, the same is true for $\mathcal{F}$ by Lemma 15 of [22, p.18]. Part11 now follows from Theorem 21 of [22, p.157] together with the Equicontinuity Lemma of [22, p.150]: Since the graphs of functions from $\mathcal{F}$ form a polynomial class, the covering numbers are bounded 22 . p.34, Lemma 36] in such a way that the corresponding covering integrals fulfill the condition of the Equicontinuity Lemma.

For part 2, consider $X$ as a random Fourier series. Uniform convergence of the random Fourier series $X$ follows from sufficiently rapid convergence of the corresponding series of squared coefficients [18. We show that a necessary and sufficient condition is fulfilled. Set

$$
X^{(m)}(t)=\sum_{\substack{k \in \mathbf{Z}^{d} \\\|k\|_{2} \leq m}} \eta_{k} T_{k} e^{2 \pi i k^{\prime} t} .
$$

Since the $\eta_{k}$ are the Fourier-coefficients of $f$, the pointwise limit of $X^{(m)}$ is a Gaussian process with the same finite-dimensional distributions as $X$. Let $I_{+}$be defined as in Section 3 set $I_{-}=\left\{-k: k \in I_{+}\right\}$, and define $X_{+}^{(m)}, X_{+}$, and $X_{-}^{(m)}, X_{-}$like $X^{(m)}$ and $X$ but with the index-range $\mathbf{Z}^{d}$ replaced by $I_{+}$and $I_{-}$, respectively. Since $\left\|X-X^{(m)}\right\|_{\infty} \leq\left\|X_{+}-X_{+}^{(m)}\right\|_{\infty}+\left\|X_{-}-X_{-}^{(m)}\right\|_{\infty}$, it is sufficient to show that both $X_{+}^{(m)}$ and $X_{-}^{(m)}$ converge uniformly. For $X_{+}^{(m)}$, note that the 
$\left(T_{k}\right)_{k \in I_{+}}$are independent, and set

$$
\sigma(s)=\left(\sum_{k \in I_{+}}\left|\eta_{k}\right|^{2}\left|e^{2 \pi i k^{\prime} s}-1\right|^{2}\right)^{1 / 2}
$$

for $s \in[0,1]^{d}$. Since $\left|\eta_{k}\right|^{2}=\left|\eta_{-k}\right|^{2}$, Fourier-expansion gives $\sigma(s)=(g(0)-g(s))^{1 / 2}$. Moreover, let

$$
\begin{aligned}
m_{\sigma}(\epsilon) & =\lambda\left\{s \in[0,1)^{d}: \sigma(s)<\epsilon\right\} \quad \text { and } \\
\bar{\sigma}(u) & =\sup \left\{y \in \mathbf{R}: m_{\sigma}(y)<u\right\}
\end{aligned}
$$

where $\lambda$ denotes the Lebesgue measure. Since the first partial derivatives of $g$ are continuous and bounded, $g$ is Lipschitz-continuous on $[0,1]^{d}$, i.e., $\sigma(s) \leq c\|s\|_{\infty}$ for some positive constant $c$. This gives $\bar{\sigma}(u) \leq c u^{1 / d}$, and for

$$
I(\sigma)=\int_{0}^{1} \frac{\bar{\sigma}(u)}{u\left(\log \frac{4}{u}\right)^{1 / 2}} d u
$$

we obtain $I(\sigma)<\infty$. With Theorem 1.1 from [18 p.9], $X_{+}^{(m)}$ is uniformly convergent with probability one. Repeating this argument with $X_{-}^{(m)}$ completes the proof.

Proof of Theorem 3 Let $X=\left(\|\varrho \cdot N\|_{2}^{2}-\mu\right) / \sigma, Y=(R-\nu) / \sqrt{2 \nu}$, and let

$$
\begin{aligned}
& f_{X}(t)=e^{-i t \mu / \sigma} \prod_{k}\left(1-2 i t \varrho_{k}^{2} / \sigma\right)^{-1 / 2} \quad \text { and } \\
& f_{Y}(t)=e^{-i t \sqrt{\nu / 2}}(1-i t / \sqrt{\nu / 2})^{-\nu / 2}
\end{aligned}
$$

be the characteristic function of $X$ and $Y$, respectively. Taylor-expansion of the corresponding cumulant generating functions gives

$$
\begin{aligned}
& \log f_{X}(t)=\frac{-t^{2}}{2}-\frac{i t^{3}}{3 \sqrt{\nu / 2}}+2 t^{4} \sum_{k}\left(\frac{\varrho_{k}^{2} / \sigma}{1-2 i \eta \varrho_{k}^{2} / \sigma}\right)^{4} \quad \text { and } \\
& \log f_{Y}(t)=\frac{-t^{2}}{2}-\frac{i t^{3}}{3 \sqrt{\nu / 2}}+\frac{t^{4}}{2 \nu}(1-i \eta / \sqrt{\nu / 2})^{-4} .
\end{aligned}
$$

From the Inversion Theorem, we obtain

$$
\begin{aligned}
& 2 \pi|P(X \leq t)-P(Y \leq t)| \leq \int_{-\infty}^{\infty} \frac{1}{|t|}\left|f_{X}(t)-f_{Y}(t)\right| d t \\
& \leq \int_{|t|<T} \frac{1}{|t|}\left|f_{X}(t)-f_{Y}(t)\right| d t+\int_{|t| \geq T} \frac{1}{|t|}\left|f_{X}(t)\right| d t+\int_{|t| \geq T} \frac{1}{|t|}\left|f_{Y}(t)\right| d t \\
& =I_{1}+I_{2}+I_{3} .
\end{aligned}
$$

Since $f_{Y}(t) \neq 0$, we have $I_{1} \leq \int_{|t|<T}\left|\frac{f_{Y}(t)}{t}\right|\left|\frac{f_{X}(t)}{f_{Y}(t)}-1\right| d t$. Since $\left|e^{x}-1\right| \leq|x| e^{|x|}$, we set $\delta(t)=\log \left(f_{X}(t) / f_{Y}(t)\right)$ and get

$$
I_{1} \leq \int_{|t|<T}\left|\frac{f_{Y}(t)}{t}\right||\delta(t)| e^{|\delta(t)|} d t
$$


with

$$
\begin{aligned}
|\delta(t)| & \leq \frac{t^{4}}{2 \nu}|1-i \eta / \sqrt{\nu / 2}|^{-4}+2 t^{4} \sum_{k}\left|\frac{\varrho_{k}^{2} / \sigma}{1-2 i \eta \varrho_{k}^{2} / \sigma}\right|^{4} \\
& \leq t^{4}\left(\frac{1}{2 \nu}+\frac{2 \sum_{k} \varrho_{k}^{8}}{\sigma^{4}}\right) .
\end{aligned}
$$

Set $c=\left(\frac{1}{2 \nu}+\frac{2 \sum_{k} \varrho_{k}^{8}}{\sigma^{4}}\right)$ and choose $T$ such that $c T^{4}=2 / 3$. We thus obtain a computable estimate for $I_{1}$ :

$$
\begin{aligned}
I_{1} & \leq 2 \int_{0}^{T}|1-i t / \sqrt{\nu / 2}|^{-\nu / 2} c t^{3} e^{c t^{4}} d t=2 \int_{0}^{T} \frac{c t^{3}}{\left(1+2 t^{2} / \nu\right)^{\nu / 4}} e^{c t^{4}} d t \\
& \leq 2 c e^{c T^{4}} \int_{0}^{T} \frac{t^{3}}{\left(1+2 t^{2} / \nu\right)^{\nu / 4}} d t \\
& =2 c e^{c T^{4}} \frac{\nu^{2} / 2-\left(1+2 T^{2} / \nu\right)^{-\nu / 4}\left(T^{4}(\nu / 2-2)+(\nu / 2)^{2}\left(T^{2}+2\right)\right)}{(\nu / 2-4)(\nu / 2-2)}
\end{aligned}
$$

Note that, for $\nu / 2=2$, the upper bound becomes $I_{1} \leq 2 c e^{c T^{4}}\left(T^{2}-2 \log \left(1+T^{2} / 2\right)\right)$, while, for $\nu / 2=4$, it becomes $I_{1} \leq 16 c e^{c T^{4}}\left(\log \left(1+T^{2} / 4\right)-T^{2} /\left(4+T^{2}\right)\right)$. With the choice of $T$, we have $e^{c T^{4}}=e^{2 / 3}$. For $I_{2}$, we set $\varrho_{*}=\max \left\{\varrho_{k}: k \geq 1\right\}$ and denote the number of occurrences of $\varrho_{*}$ in the sequence $\varrho_{1}, \varrho_{2}, \ldots$ by $K$. With this, we obtain that

$$
\begin{aligned}
I_{2} & \leq \int_{|t|>T} \frac{1}{|t|}\left|1-2 i t \varrho_{*}^{2} / \sigma\right|^{-K / 2} d t \\
& =\int_{|t|>T} \frac{1}{|t|}\left(1+4 t^{2}\left(\varrho_{*}^{2} / \sigma\right)^{2}\right)^{-K / 4} d t \\
& =\frac{4}{K}\left(2 T \varrho_{*}^{2} / \sigma\right)^{-K / 2} H_{2}\left(K / 4, K / 4, K / 4+1,-\left(2 T \varrho_{*}^{2} / \sigma\right)^{-2}\right),
\end{aligned}
$$

where $H_{2}$ denotes the second hypergeometric function. Similarly, for $I_{3}$, we obtain

$$
I_{3} \leq 2(\nu / 2)^{\nu / 4-1} T^{-\nu / 2} H_{2}\left(\nu / 4, \nu / 4, \nu / 4+1,-T^{-2} \nu / 2\right) .
$$

If we denote the thus derived bounds of $I_{1}, I_{2}$ and $I_{3}$ by $B_{1}, B_{2}$, and $B_{3}$, respectively, and set

$$
B=\left(B_{1}+B_{2}+B_{3}\right) /(2 \pi),
$$

we obtain $|P(X \leq t)-P(Y \leq t)| \leq B$.

Proof of Theorem 4. Let $\varrho^{(-m)}=\varrho-\varrho^{(m)}$ where the difference is evaluated pointwise. Since $\varrho^{(m)} \cdot C$ and $\varrho^{(-m)} \cdot C$ are independent, and since $\|\varrho \cdot C\|_{\infty}=\max \left\{\| \varrho^{(m)}\right.$. $\left.C\left\|_{\infty},\right\| \varrho^{(-m)} \cdot C \|_{\infty}\right\}$, we have

$$
\begin{aligned}
0 & \leq P\left(\left\|\varrho^{(m)} \cdot C\right\|_{\infty} \leq t\right)-P\left(\|\varrho \cdot C\|_{\infty} \leq t\right) \\
& =P\left(\|\varrho \cdot C\|_{\infty}>t\right)-P\left(\left\|\varrho^{(m)} \cdot C\right\|_{\infty}>t\right) \\
& =P\left(\|\varrho \cdot C\|_{\infty}>t \text { and }\left\|\varrho^{(m)} \cdot C\right\|_{\infty} \leq t\right) \\
& =P\left(\left\|\varrho^{(-m)} \cdot C\right\|_{\infty}>t \text { and }\left\|\varrho^{(m)} \cdot C\right\|_{\infty} \leq t\right) \\
& =P\left(\left\|\varrho^{(m)} \cdot C\right\|_{\infty} \leq t\right) P\left(\left\|\varrho^{(-m)} \cdot C\right\|_{\infty}>t\right) .
\end{aligned}
$$


Setting $\alpha_{p}=E\left(C_{1}^{p}\right)$, we obtain

$$
P\left(\left\|\varrho^{(-m)} \cdot C\right\|_{\infty}>t\right) \leq P\left(\sum_{k>m}\left|\varrho_{k}\right|^{p} C_{k}^{p}>t^{p}\right)=\frac{\alpha_{p}}{t^{p}} \sum_{k>m}\left|\varrho_{k}\right|^{p} .
$$

Since $m \geq p$,

$$
\begin{aligned}
P\left(\left\|\varrho^{(m)} \cdot C\right\|_{\infty} \leq t\right) & \leq \prod_{k=1}^{p} P\left(C_{k} \leq \frac{t}{\left|\varrho_{k}\right|}\right)=\prod_{k=1}^{p}\left(1-e^{-t /\left|\varrho_{k}\right|}\right) \\
& =\prod_{k=1}^{p} e^{-t /\left|\varrho_{k}\right|}\left|e^{t /\left|\varrho_{k}\right|}-1\right| \\
& \leq \prod_{k=1}^{p} e^{-t /\left|\varrho_{k}\right|} \frac{t}{\left|\varrho_{k}\right|} e^{t /\left|\varrho_{k}\right|}=t^{p} \prod_{k=1}^{p} \frac{1}{\left|\varrho_{k}\right|}
\end{aligned}
$$

The result follows from (12) and (13).

\section{ACKNOWLEDGMENTS}

I would like to thank Peter Hellekalek for fruitful discussions and his support, and Jürgen Eichenauer-Herrmann as well as the anonymous referees for their remarks and comments.

\section{REFERENCES}

[1] M. J. Buckley and G. K. Eagleson. An approximation to the distribution of quadratic forms in normal random variables. Austral. J. Statist., 30A:150-159, 1988.

[2] R. R. Coveyou and R. D. MacPherson. Fourier analysis of uniform random number generators. J. Assoc. Comput. Mach., 14:100-119, 1967. MR 36:4779

[3] R. Cranley and T. N. L. Patterson. Randomization of number theoretic methods for multiple integration. SIAM J. Numer. Anal., 13:904-914, 1976. MR 58:13605

[4] R. W. Farebrother. The distribution of a quadratic form in normal variables. J. Royal Statist. Soc. C, 39:294-309, 1990.

[5] H. Faure. Discrépance et diaphonie en dimension un. In Advances in number theory (Kingston, ON, 1991), Oxford Sci. Publ., pages 411-419. Oxford Univ. Press, New York, 1993. MR 97d:11115

[6] K. Frank and S. Heinrich. Computing discrepancies related to spaces of smooth periodic functions. In Monte Carlo and quasi-Monte Carlo methods 1996 (Harald Niederreiter et al., eds.), Lecture Notes in Stat., Vol. 127, pages 238-250. Springer, New York, 1997. MR 99g:65026

[7] V. S. Grozdanov. On the diaphony of two-dimensional finite sequences. C. R. Acad. Bulgare Sci., 48:15-18, 1995. MR 97d:11116

[8] S. Heinrich. Efficient algorithms for computing the $L_{2}$-discrepancy. Math. Comp., 65:16211633, 1996. MR 97a:65024

[9] P. Hellekalek. On the assessment of random and quasi-random point sets. In Random and quasi-random point sets (Peter Hellekalek et al., eds), Lecture Notes in Stat., Vol. 138, pages 49-108. Springer, New York, 1998. MR 2000c:11127

[10] P. Hellekalek and H. Leeb. Dyadic diaphony. Acta Arith., 80:187-196, 1997. MR 98g:11090

[11] P. Hellekalek and H. Niederreiter. The weighted spectral test: diaphony. ACM Trans. Modeling and Computer Simulation, 8:43-60, 1998.

[12] F. J. Hickernell. A generalized discrepancy and quadrature error bound. Math. Comp., 67:299-322, 1998. MR 98c:65032

[13] F. J. Hickernell. Lattice rules: how well do they measure up? In Random and quasi-random point sets (Peter Hellekalek et al., eds.), Lecture Notes in Stat., Vol. 138, pages 109-166. Springer, New York, 1998. MR 2000b:65007 
[14] J. Hoogland and R. Kleiss. Discrepancy-based error estimates for quasi-Monte Carlo. I: general formalism. Comp. Phys. Comm., 98:111-127, 1996.

[15] J. Hoogland and R. Kleiss. Discrepancy-based error estimates for quasi-Monte Carlo. II: results for one dimension. Comp. Phys. Comm., 98:128-136, 1996.

[16] H. Leeb. Weak limits for diaphony. In Monte Carlo and quasi-Monte Carlo methods 1996 (Harald Niederreiter et al., eds.), Lecture Notes in Stat., Vol. 127, pages 330-339. Springer, New York, 1997. MR 99i:11062

[17] J. J. Liang, K. T. Fang, F. J. Hickernell, and R. Z. Li. Testing multivariate uniformity and its applications. Math. Comp. 70:337-355, 2001. MR 2001f:62032

[18] M. B. Marcus and G. Pisier. Random Fourier series with applications to harmonic analysis, volume 101 of Annals of Mathematics Studies. Princeton University Press, Princeton, N.J., 1981. MR 83b:60031

[19] A. B. Owen. Randomly permuted $(t, m, s)$-nets and $(t, s)$-sequences. In Monte Carlo and quasi-Monte Carlo methods in scientific computing (Harald Niederreiter et al., eds.), Lecture Notes in Stat., Vol. 106, pages 299-317. Springer, New York, 1995. MR 97k:65013

[20] A. B. Owen. Monte Carlo variance of scrambled equidistribution quadrature. SIAM J. Numer. Anal., 34:1884-1910, 1997. MR 98j:65006

[21] A. B. Owen. Scrambled net variance for integrals of smooth functions. Ann. Stat., 25:15411562, 1997. MR 98j:65004

[22] D. Pollard. Convergence of Stochastic Processes. Springer Series in Statistics. Springer, New York, 1984. MR 86i:60074

[23] A. van Hameren, R. Kleiss, and J. Hoogland. Gaussian limits for discrepancies. I: Asymptotic results. Comp. Phys. Comm., 107:1-20, 1997. MR 99k:65009

[24] P. Zinterhof. Über einige Abschätzungen bei der Approximation von Funktionen mit Gleichverteilungsmethoden. Sitzungsber. Österr. Akad. Wiss. Math.-Natur. Kl. II, 185:121-132, 1976. MR 58:19037

[25] P. Zinterhof and H. Stegbuchner. Trigonometrische Approximation mit Gleichverteilungsmethoden. Stud. Sci. Math. Hungar., 13:273-289, 1978. MR 83a:10088

Department of Statistics, University of Vienna, Universitätsstrasse 5, A-1010 ViENNA, Austria

E-mail address: hannes.leeb@univie.ac.at 